\title{
Application of supercapacitor to power small electronic appliances
}

\author{
M Das $^{1}$, I.Das ${ }^{2}$, N.K.Bhattacharyya ${ }^{3}$, D.Mukherjee ${ }^{4}$, H.Saha $^{5}$ \\ ${ }^{I}$ (AEIE Department, Haldia Institute of Technology, W.B, India) \\ ${ }^{2,5}$ (CEGESS, Bengal Engineering and Science University, W.B, India) \\ ${ }^{3}$ (Metra Electronics, Webel Electronics Complex, P-1, Taratala Road, W.B, India) \\ ${ }^{4}$ (ETCE Department, Bengal Engineering and Science University, W.B, India)
}

\begin{abstract}
The supercapacitor has been considered as a source of potential power. The charging of supercapacitor module has been established from photovoltaic module along with a constant voltage constant current converter to power Li-ion battery compatible systems. Fast charging feature of supercapacitors with a compatible photovoltaic module and a power converter is of great use in rural area, where grid power is not available, as a small community charging module can benefit multiple users. In this paper the charging of supercapacitor and discharging of it to a small electronic gadget has been considered.
\end{abstract}

Keywords - Buck converter, Charging, Discharging, Li-ion battery, Supercapacitor

\section{Introduction}

Supercapacitor has a good prospect to act as a power source as well energy storage element to power modern electronic gadgets like mobile phone. In recent scenarios, supercapacitors have been used in different consumer applications i.e., for elevators and electric buses [1],wind energy applications [2], photovoltaic powered products [3] including mobile phones [4],[5].

Present day mobile phones are the most frequently used consumer electronic devices which are also widespread in far flung areas not catered by grid power. Most of such electronic appliances use Li-ion battery as a power source which does not ensure long cycle life. The reported cycle life of Li-ion battery and supercapacitor are 400-1200 load cycles and 500,000 [6] load cycles respectively. The high cycle life of a supercapacitor as compared to Li-ion battery thus offer benefit to the users. Real challenge lies in the design of charging and discharging circuit in order to ensure operation of supercapacitor within safe limits and delivery of battery compatible output. A comparison between supercapacitor and Li-ion battery is given in TABLE 1.

Table 1: Comparison between Li-Ion battery and supercapacitor

\begin{tabular}{|c|c|c|}
\hline Function & Supercapacitor & Li-ion Battery \\
\hline Charge Time & $1-10 \mathrm{sec}$ & $10-60 \mathrm{~min}$ \\
\hline Cycle life & 1 million & 500 or higher \\
\hline Cell voltage & $2.7 \mathrm{~V}$ & $3.6 \mathrm{~V}-3.7 \mathrm{~V}$ \\
\hline $\begin{array}{c}\text { Specific } \\
\text { energy(Wh/kg) }\end{array}$ & 5 & $100-200$ \\
\hline $\begin{array}{c}\text { Specific } \\
\text { power(W/kg) }\end{array}$ & Up to 10,000 & $1000-3000$ \\
\hline Cost per Wh & $\$ 20$ & $\$ 0.50-1.00$ \\
\hline
\end{tabular}

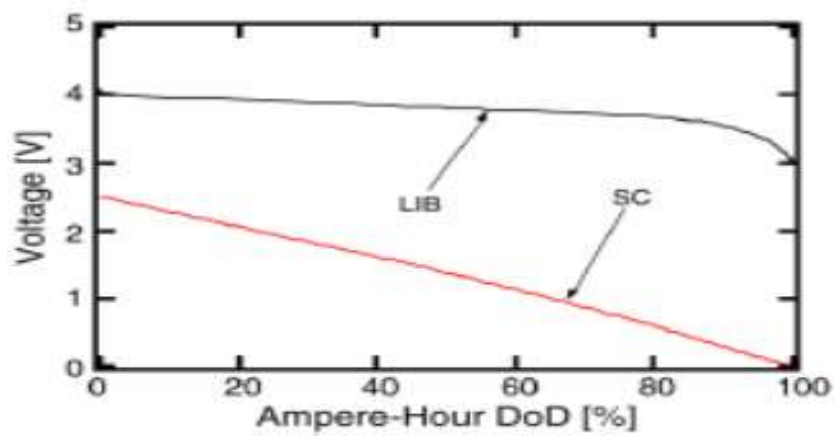

Fig. 1 Typical discharge curves of batteries and supercapacitor cells as a function of Depth of Discharge (DoD) 
The supercapacitor versus Li-ion battery discharge characteristics [7] is given in Fig. 1. From Fig. 1, it is evident that battery delivers $3.2 \mathrm{Wh}$ on reaching $80 \%$ Depth of Discharge (DoD). The supercapacitor chosen by the authors is 4 numbers each $350 \mathrm{~F}, 2.7 \mathrm{~V}$ connected in series operating from a pedestal of $10 \mathrm{~V}$ down to $5 \mathrm{~V}$ level shall deliver $1 \mathrm{Wh}$. The user requirement can still be met with increased charging frequency in view of much higher charge - discharge cycle life of supercapacitor.

Higher charging and discharging efficiency of supercapacitor justifies the use of buck converter to obtain the constant voltage to make it compatible with a battery. In this paper a low cost power converter unit is proposed in conjunction with a compatible PV module as a charging unit for supercapacitor for use in remote areas. Separate sections have been devoted to illustrate the charging and discharging operations along with performance analysis.

\subsection{Charging of supercapacitor bank from PV module}

\section{Design}

A suitable converter as given in Fig. 2 was designed to charge the supercapacitor module of $10.8 \mathrm{~V}$ consisting of four series connected 350F, 2.7V (Maxwell Technologies made) supercapacitor elements. As a part of rudimentary design consideration we have chosen four times voltage in order to ensure $75 \%$ of the stored energy availability at output. We have chosen a buck converter which suits available output of industry standard 12 V PV modules.

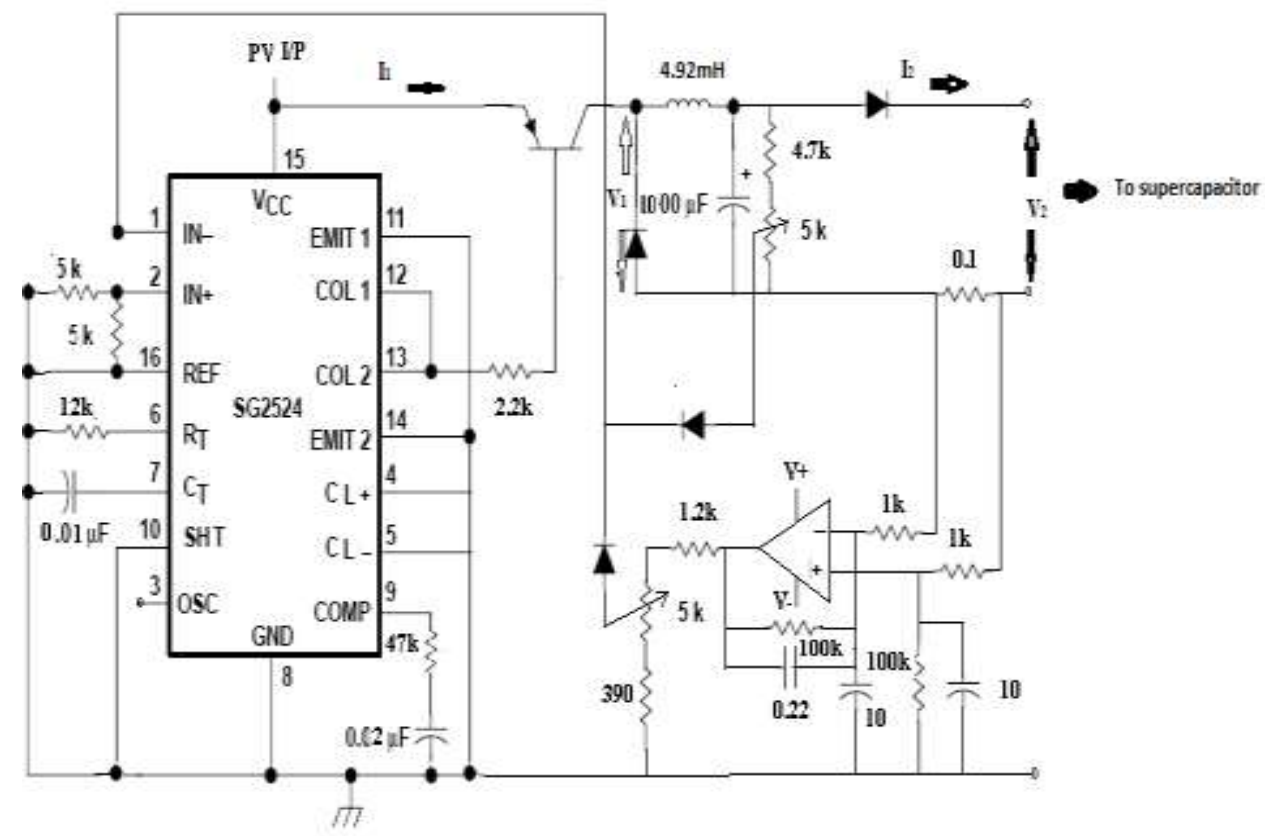

Fig. 2 The designed buck converter unit.

Block diagram of total charging scheme is shown in Fig. 3

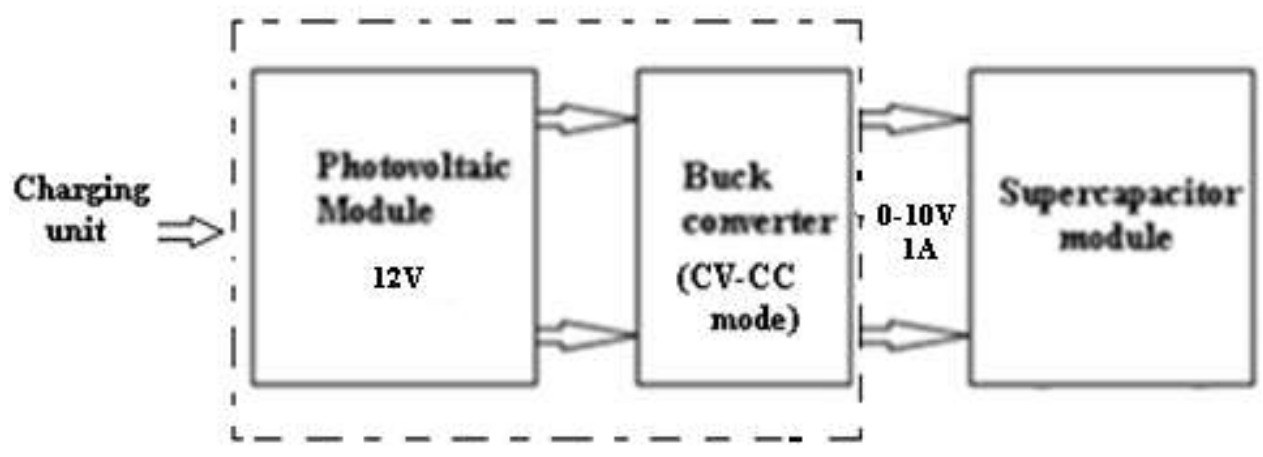

Fig. 3 The schematics of charging of supercapacitor module 
Table 2: Performance characteristics of the designed buck converter

\begin{tabular}{|c|c|c|c|c|c|}
\hline $\begin{array}{c}\text { Time } \\
(\mathbf{s e c})\end{array}$ & $\begin{array}{c}\text { Photovoltaic I/P } \\
\text { voltage } \\
(\mathbf{V})\end{array}$ & $\begin{array}{c}\text { Voltage across } \\
\text { supercapacitor module (V) }\end{array}$ & $\begin{array}{c}\mathbf{O} / \mathbf{P} \\
\text { Power } \\
(\mathbf{W})\end{array}$ & $\begin{array}{c}\mathbf{I} / \mathbf{P} \\
\text { Power } \\
(\mathbf{W})\end{array}$ & $\begin{array}{c}\text { Charging } \\
\text { Efficiency } \\
(\mathbf{\%})\end{array}$ \\
\hline 2 & 16.5 & 0.25 & 0.25 & 0.64 & 39 \\
\hline 34 & 16.15 & 0.6 & 0.6 & 0.968 & 62 \\
\hline 53 & 15.89 & 0.9 & 0.9 & 1.1925 & 75 \\
\hline 74 & 15.66 & 1.2 & 1.2 & 1.5 & 80 \\
\hline 98 & 15.3 & 1.65 & 1.65 & 2.1 & 78 \\
\hline 133 & 15 & 2.1 & 2.1 & 2.574 & 81 \\
\hline 169 & 14.73 & 2.5 & 2.5 & 3.29 & 76 \\
\hline 205 & 14.45 & 3 & 3 & 3.72 & 80 \\
\hline 287 & 14.22 & 4 & 4 & 4.76 & 84 \\
\hline 372 & 13.9 & 5 & 5 & 5.916 & 84 \\
\hline 460 & 13.9 & 6 & 6 & 6.9165 & 86 \\
\hline 555 & 13.69 & 7 & 6.86 & 7.992 & 86 \\
\hline 655 & 13.61 & 8 & 7.6 & 9.0804 & 83 \\
\hline 705 & 13.75 & 8.5 & 7.65 & 9.315 & 82 \\
\hline 758 & 14.4 & 9 & 7.65 & 8.268 & 92 \\
\hline 803 & 16.6 & 9.25 & 3.7 & 3.952 & 93 \\
\hline
\end{tabular}

It is evident from the TABLE 2 that the charging converter efficiency is better than $80 \%$ during operation from $20 \%$ of rated output to rated capacity. The converter has constant voltage output with protective current limits. The charge time characteristics as obtained have been given in Fig. 4.

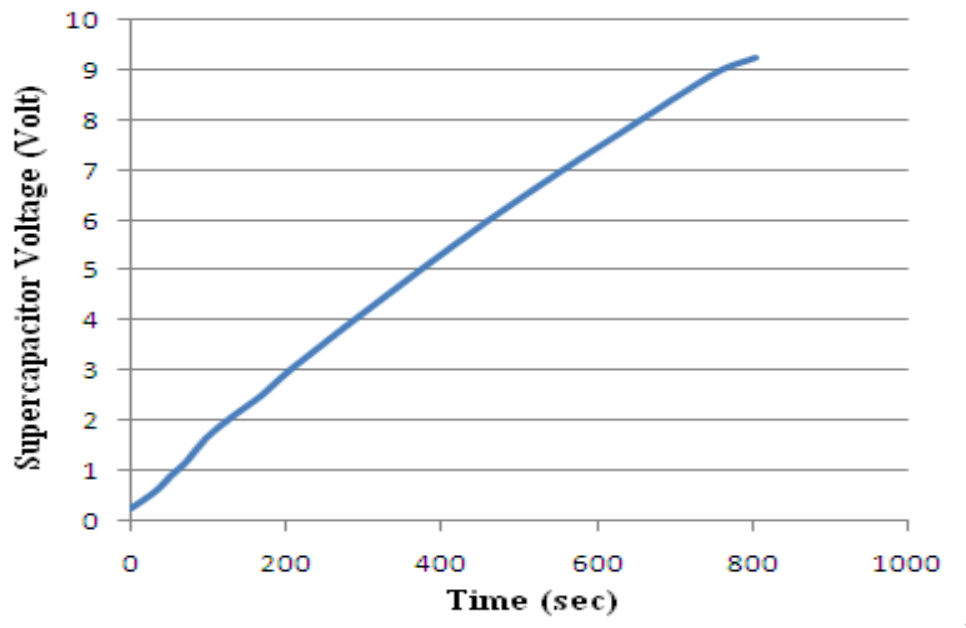

Fig. 4 The charge time characteristics of supercapacitor module

As shown in Fig. 4, the charging time of few hundred seconds offers scope to use of PV module along with the charge converter scheme described above as community charging system in rural areas to multiple users. There are three types of popular charging topologies. They are constant voltage (CV) charging, constant power (CP) charging and constant current $(\mathrm{CC})$ charging. Constant voltage $(\mathrm{CV})$ charging is not compatible here due to variation in voltage encountered at capacitor terminals during charging. Constant power (CP) charging is more complex and high charge current at the beginning might be detrimental to capacitor due to higher losses at capacitor. In this paper a constant voltage and constant current mode (CV-CC) of charging has thus been used to charge the capacitor bank from PV voltage source. A constant current of 1A is selected to suit the time available for charging. The test bench during the charging of supercapacitor has been shown in Fig. 5 . 


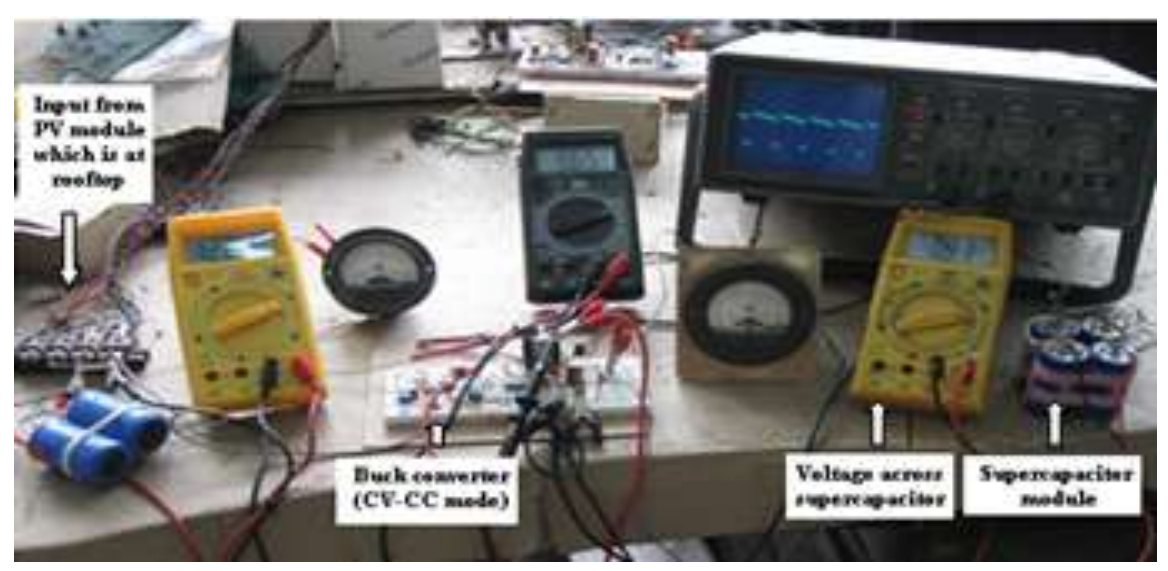

Fig. 5 The test bench to charge a supercapacitor module from solar PV module.

\subsection{Discharging of supercapacitor module}

There is ample scope to use an appropriate buck converter to get desirable constant voltage profile as obtained from battery. Here, 34063A chip is used to transfer the supercapacitor energy for powering the gadget. The necessary hardware along with the scheme is shown in Fig. 6.

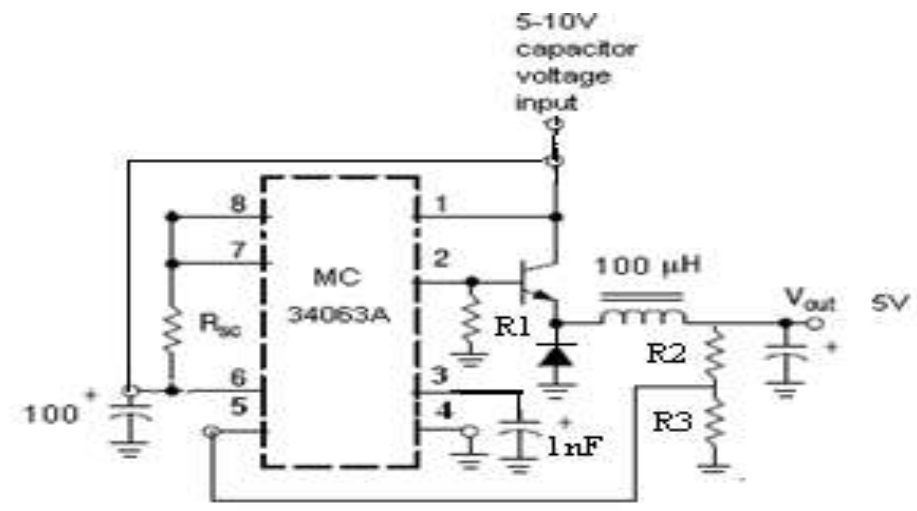

Fig. 6 The circuit using 34063A chip to act as power converter in CV mode.

\section{Proposed Energy Storage with Charging and Discharging Facility}

The scheme employing community PV module and a buffer unit comprising of supercapacitor bank integrated with charging circuit and output converter has been indicated as in Fig. 7.

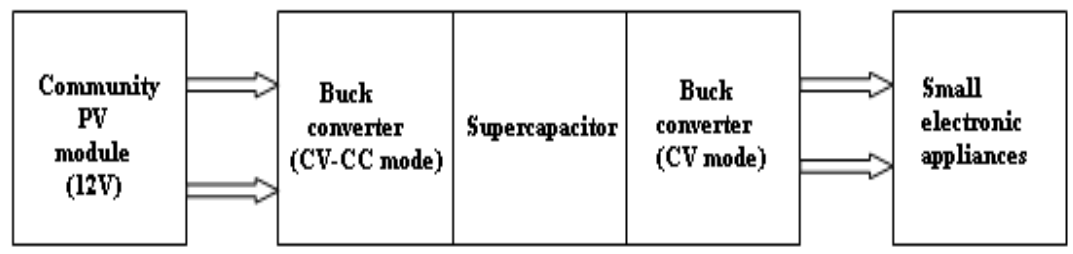

Fig. 7 The block diagram of proposed energy storage with charging and discharging facility

\section{Conclusion}

It can be seen that using supercapacitors in buffer mode offer a good potential for rural users who are not connected by utility grid. Thus the proposed PV module based community charging system can benefit multiple users from the long life of supercapacitor power source coupled with fast energy absorption property of supercapacitor as compared to use of battery to power the small electronic appliances. The designed circuit has given promising results in respect of fast charging and meeting the required energy usage. Still there is ample scope to investigate optimum charge and discharge protection possibilities for the elements of the capacitor bank. 


\section{References}

[1] P. Barrade and A. Rufer, Supercapacitors as energy buffers as a solution for elevators and for electric busses supply, Proc. Power Conversion Conference, Osaka, 2002, 1160-1165.

[2] C. Abbey and G. Joes, Supercapacitor energy storage for wind energy applications, IEEE Transactions on Industry Applications, 43(3), 2007, 769-776.

[3] S.Y. Kan, M. Verwaal and H. Broekhuizen, Battery - capacitor combinations in photovoltaic powered products, Journal of Power Sources, 162(2) 2006, 971-974.

[4] J. Monteiro, N. Garrido and R. Fonseca, Efficient supercapacitor energy usage in mobile phones, IEEE International Conference on Consumer Electronics, Berlin, 2011, 318-321.

[5] L. Palma, P. Enjeti and J.W. Howze, An approach to improve battery run time in mobile applications with supercapacitors, Power Electronics Specialist Conference, 2, 2003,918-923.

[6] V.A. Shah, J.A. Joshi, R. Maheshwari, and R. Roy, Review of ultracapacitor technology and its applications, Proc. National Power Systems Conference, Bombay, 2008, 142-147

[7] M. Uno, and K. Tanaka, Accelerated charge-discharge cycling test and cycle life prediction model for supercapacitors in alternative battery applications, IEEE Transactions on Industrial Electronics, 59(12), 2012, 4704 - 4712. 